\title{
Young Adults with Diabetes Discuss Barriers and Solutions to Diabetes Management
}

\author{
SeAnne Safaii ${ }^{1, *}$, Martha Raidl ${ }^{1}$, Samantha Ramsay ${ }^{2}$ \\ ${ }^{1}$ College of Agriculture and Life Sciences, University of Idaho, Boise, 83702, Idaho, United States \\ ${ }^{2}$ College of Agriculture and Life Sciences, University of Idaho, Moscow, 83844, Idaho, United States \\ *Corresponding Author: sjsafaii@uidaho.edu
}

Copyright $@ 2013$ Horizon Research Publishing All rights reserved.

\begin{abstract}
The purpose of this study was to identify perceived barriers young adults experience in diabetes self-management and possible strategies to overcome these barriers. Twenty two young adults, 18-28 years- old, with type 1 or type 2 diabetes attended three focus groups held in two north western states. Participants were asked to discuss barriers and strategies to help them manage their diabetes. The transcripts were coded and analyzed for themes. Four themes were identified: 1) lack of understanding of diet principles, medications and physical activity; 2) lack of support from family, friends and health care providers; 3 ) lack of resources to assist in managing blood sugar levels; and 4) participants reported the use of the internet and social networking as strategies for overcoming barriers. There are not a lot of materials developed for this age group. Most diabetes educational materials focus on children or adults. This qualitative study provides important insights from young adults regarding the development of educational materials and support systems to help them successfully manage their diabetes.
\end{abstract}

Keywords Diabetes, Young Adults, Barriers, Solutions, Management

\section{Introduction}

An estimated 25.8 million Americans or $8.3 \%$ of the population have diabetes [1]. Unfortunately, the incidence of diabetes is increasing most rapidly in children and young adults. The Centers for Disease Control and Prevention (CDC) estimates that $3.7 \%$ of $20-44$ year-olds have diabetes and most of these cases are type 2 diabetes [1]. The cost of diabetes is staggering. A recent report published by the American Diabetes Association (ADA) estimates \$245 billion was spent on direct and indirect costs incurred by people with diabetes in 2012 [2]. The young adult population with diabetes is increasing. Due to the challenges of transitioning from adolescence to young adulthood, this age group has a heightened need for diabetes services and support. Providing age appropriate behavioral recommendations is necessary for improving glycemic control, quality of life, and avoiding long term complications [3].

Diabetes management includes following a diabetes meal plan, being physically active and possibly taking medication. Individuals with diabetes who don't keep their blood sugar under control suffer long-term complications that include nephropathy, neuropathy, and retinopathy. Young adults with diabetes are more likely to exhibit these complications at an earlier age [4], [5], [6], have a shortened life span of 17-18 years [1] and a three-fold to six-fold increased mortality, compared with the general population [7]. The transition from pediatric to adult health care often results in a loss of patient follow-up, which is especially detrimental to young adults who begin to experience early signs of diabetic complications [8].

Two major obstacles prevent young adults with diabetes from being able to manage their diabetes. The first is a lack of programs for young adults. Most diabetes programs focus on younger children (those with type 1 diabetes) or older adults (those with type 2 diabetes) that are over 40 years-old. The second obstacle is the loss of family and medical support systems that helped them monitor their diabetes when they transition from living at home to living away from family. These transitions can include going away to school, entering the workplace, getting married, and having children. As a result, they no longer receive family and medical support or health insurance from their parents [1], [9], [10], [11].

Young adults are responsible for their own self-management and providers will often ask them to describe their experiences and reactions on their current metabolic control as well as have them set their own goals to make changes in regimens for management [12]. Considering the expected responsibilities for young adults who are transitioning to self-care, the Expanded Health Belief Model (EHBM) was used as the theoretical framework in understanding the perceptions of young adults' diabetes management. The EHBM is the most frequently used Social Cognition Model to predict health behaviors. The model suggests that behavior is influenced by an 
individual's beliefs and attitudes related to health outcomes . Therefore, there were two objectives of this study. The first was to determine perceived beliefs about barriers to diabetes self-management for young adults. The second was to identify strategies to help them manage their diabetes. These strategies would then be incorporated into an education curriculum designed for this age group. Questions for the focus groups were based upon the EHBM and specifically asked them to discuss barriers and strategies to their diabetes self-management [12].

The following methods were designed to gather detailed information regarding this understudied group.

\section{Materials and Methods}

\subsection{Participants}

Twenty two young adults' ages 18-28 years-old participated in three focus groups. Table 1 lists the demographic characteristics of the group. Sixteen had type 1 diabetes, and six had type 2 diabetes. Nine were male and thirteen were female. All participants were Caucasian. Participants were not segregated by diabetes type due to the small number of participants in each focus group and because the researchers felt the dialogue between the two groups would add richness to the focus group discussion. In addition, it was important to know if both diabetes groups had similar barriers and strategies and if one diabetes curriculum could be designed for both diabetes groups.

\subsection{Procedure}

The university Institutional Review Board approved the methodology for this study. Using focus group guidelines from Krueger and Casey [13], the three researchers developed the questions after meeting with, young adults with diabetes and a diabetes health care team. An expert panel of seven diabetes educators/health care professionals reviewed and approved three questions, which were pilot tested. The following three questions and subsequent probes, addressed focus group participants' barriers and strategies to managing their diabetes and covered diet, medications, and physical activity:

1) What are the barriers to managing your diabetes diet and how do you overcome these barriers?

2) What are the barriers to managing your diabetes medication(s) and how do you overcome these barriers?

3) What are the barriers to physical activity and how do you overcome these barriers?

Participants were recruited as volunteers from diabetes education programs using flyers, phone calls and letters. They were between 18-28 years-old and had type 1 or type 2 diabetes. They completed a consent form, and received a $\$ 50$ stipend to offset the costs of travel and child care.

\subsection{Focus Group Discussions}

Focus groups are used to collect data regarding a particular phenomenon through the use of qualitative research methodology [13].In-depth information was obtained from participants by having them respond spontaneously and creatively to open-ended questions. Appropriate probes were used to elicit further responses. Use of this methodology allowed for participants' perceptions to be gathered, which cannot be obtained from a written survey. A purposive sample of five to ten participants attended each focus group session.

Three researchers, one lead and two assistants, conducted the focus groups. The lead researcher moderated the three focus group discussions, which were audio recorded and professionally transcribed. The two assistants recorded field notes to supplement the audio recordings. Each focus group lasted approximately 90 minutes.

\subsection{Data Collection and Analysis}

Initially the three researchers met and used an open coding system to group similar transcribed information into broad categories. This was later replaced with more selective codes or themes that were tied to a core concept. Each researcher independently assigned participants' responses to an appropriate theme, which were then counted using a qualitative data software called Ethnograph (v5) [14].

Duplicate statements by participants in the same focus group were counted only once. For example, if two participants from the same focus group stated that they could not afford glucose strips, this statement was only counted once. Therefore, only differing statements were placed in themes and used for analysis, providing a measure of depth to the discussions. Themes that contained less than $5 \%$ or fewer responses were eliminated due to their minimal impact on the results. The independently coded statements from the three researchers had an inter-rater reliability agreement of $82 \%(\mathrm{r}=0.82)$ on barriers to diabetes self-management and $78 \% \quad(\mathrm{r}=0.78)$ for strategies on overcoming barriers to management of their diabetes. Inter-rater reliability of $70 \%$ $(r=0.70)$ or higher is considered reliable [15].

\section{Results}

\subsection{Themes}

After analyzing participant responses, four major themes emerged which are listed in Table 2: 1) lack of understanding about diabetes principles related to diet, medication, and physical activity, 2) lack of social and medical support, 3) lack of resources, and 4) participant reported strategies for overcoming barriers

\section{Lack of Understanding}

An overarching theme from the focus group interviews was a lack of participants' understanding of their diabetes management related to diet principles, medications, and physical activity recommendations. Diet principles that 
participants didn't understand were: carbohydrate counting, calculating calories, recognizing portion sizes, and managing social settings where alcohol was present. In describing calorie counting, one participant stated, "I've done it [calorie counting] for 17 years and it's still sometimes a little iffy." Another participant described the challenges with managing portions and implementing carbohydrate counting: "It's just not feasible to carry around a scale or a measuring cup or one of those books [carbohydrate counting] to know exactly how much you're gonna eat."

Participants described their own lack of understanding of the medications to control their diabetes, by stating they didn't know how often they should be testing their blood glucose levels and how to treat high and low blood sugar levels.

Understanding how to manage blood glucose levels before, during and after physical activity was even more challenging. One participant described challenges with determining when and how much to eat when he engages in an afternoon game of softball, stating, "So I'll have a game at 2:00 and I can't eat lunch. I can't eat till after the double header."

Diabetes is a complicated chronic disease. Therefore it was not surprising participants reported a lack of understanding on how to manage their diet, medication, and physical activity. This lack of understanding could have been due to not assuming responsibility for their diabetes, and relying on family, friends, and health care providers to manage their diabetes. Parents of children with diabetes tend to be overprotective [16] and health care professionals tend to direct information to their parents and exclude them in the education session. Coyne \& Gallagher [17] found children want to be included in discussions about their diabetes. Including them in decisions about their care could help them develop their decision-making capabilities and help them manage their diabetes as they grow.

The specific comments participants made about their diabetes diet could be due to the diet being the most difficult diabetes self-management behavior to follow [18], [19], as well as the myriad of decisions they have to make on what to eat, when to eat, and how much to eat [20]. In contrast, the general comments about lack of understanding for medication and physical activity focused on the role these two components played in keeping their blood sugars levels under control.

\section{Lack of Support}

The second theme identified from participants' responses in their diabetes management was the lack of support from family and friends, as well as health care providers. There were three reasons why they felt a lack of support from family and friends: (1) these individuals had a very limited knowledge or understanding about diabetes, (2) they were no longer reminding them to check blood sugars, and (3) they didn't understand the physical disabilities related to their diabetes. One participant stated "family and friends don't understand 'cause they don't have it[diabetes]". Another participant described a family member's response when his blood glucose fell below 23, "we just got glucagon kits and she didn't know what to do." Not having Mom's support to remind them to test blood sugars was especially difficult. "It's really hard to test your blood sugar. It's just the last thing on your list and Mom's not yelling at you." Some participants had physical disabilities, arthritis, injured knees or back, kidney problems, and found that this made it difficult for family and friends to help them.

Feeling a lack of support from health care providers was based on the negative responses they received from doctors/nurses/dietitians and conflicting information they received if they saw more than one health care provider. "They (physicians) have nothing to do with each other and they don't really work together a whole lot-the gastroenterologists and the endocrinologists. It would be nice to talk to them at the same time so that they know what each one is telling you and they can correspond. I don't see why there wouldn't be a dietitian in there, but having two contradicting diets, it'd be nice to actually talk to somebody who might be able to work something out."

As adults, our participants experienced lack of support from two groups- family/friends and the medical community - and felt isolated. Several researchers have found support from family, and strong relationships help promote self-care behaviors and improve diabetes management, which could offset this lack of support from family/friends and health care providers [9], [21], [19].

The criticism participants made of the health care providers could be due to various factors. The first is how the patient-doctor session is conducted. Some researchers have found that many doctors use confusing medical and technical jargon as well as interrupting patients and dominating the session [22]. The second is that patients feel less motivated to follow recommendations when they are scolded by a provider [23]. Furthermore, Anderson [24] speculated that when disagreements between doctor and patient over expectations for diabetes occur, patient anxiety increases which results in avoidance of self-care.

\section{Lack of Resources}

A lack of resources was the third theme participants identified that related to their diabetes management. Three resources that were limited included a lack of access to health care providers, lack of access to diabetes materials, and lack of funds. Many participants did not have health insurance. As two participants explained, "My biggest barrier is the need for health care reform. It (diabetes) is your whole life, man." As a result, many felt unable to get questions answered immediately, especially at critical times. One participant mentioned how he felt when his blood sugar levels were over 200 and he needed immediate assistance, "it feels like you're carrying like 50 pound weight on you and you're trying to run and you can't play to your full potential and I think that really sucks." They mentioned that easy to understand materials on diabetes medication would be helpful.

A lack of funding resources presented challenges as well. Many participants stated their limited funds did not cover the high costs for medications and testing (i.e. test strips, 
laboratory tests). "You can't do anything else 'cause you're just paying to be diabetic," and "I'm on a broken pump right now because we can't afford a new one."

Lack of access to medical providers [25], lack of a health care finance system, lack of funds and lack of access to educational materials prevent diabetes patients from having continuity of care as they transition to an adult model of medical care [3]. Lack of funds and the costs and resulting stress associated with having diabetes are serious problems. The average cost of diabetes care for a person with diabetes can exceed $\$ 20,000$ per year in out-of-pocket costs for routine doctor visits, lab work and diabetes medications and blood glucose checking supplies. This figure does not include hospitalizations or the purchase of an insulin pump which is approximately $\$ 6,000$ [26].

\section{Strategies to Manage Diabetes}

Participants provided strategies for overcoming their identified barriers to managing their diabetes. To overcome the lack of understanding barriers in their diabetes self-management, they suggested designing educational materials that focused on diet and medications and were easy to access. They felt these materials should include "visual graphics that would explain diet principles" plus "recipes and menu planning information." They wanted medication information to be online and simple to understand, with a focus on the basics, i.e. when they should test their blood glucose levels, and how they and their family should treat high and low blood sugar levels. Participants did not provide any strategies to help them meet physical activity recommendations.

To overcome lack of support, participants suggested technology strategies to take the place of family/friends and health care providers. To remind them to check and track blood sugars, they suggested using Smart phones, Facebook, Ipad, computer, and Apps. "So I think that would be cool for diabetics to [post] blood sugar status and you could comment on it," on Facebook. Furthermore, an online diabetes support group would allow them to share the effects of certain foods, and medications (like pumps and insulin shots) had on their blood sugar levels. They would prefer to interact with an online or virtual health care provider support group instead of receiving criticisms in a face-to-face environment.

Strategies for overcoming lack of resources addressed the issue with their lack of access to health care providers and they suggested information was in an online information center. In this online center, they would submit questions on diet and blood sugars and receive instant feedback. "I think it would be a lot of help for the doctors too. To be able to get a daily update instead of waiting a week or a month or three months to tell them what you've been eating, a daily update versus your blood sugars, what you're eating, and how you feel. It'd probably help them a lot more to understand their individual patients." Their strategy for overcoming the costs associated with diabetes was universal health care.

Findings on strategies for overcoming barriers to physical activity were inconclusive because there were fewer than 5\% similar responses in the strategies category, so they were eliminated. Even though the facilitator probed repeatedly, participants at all three sites did not engage in their discussion of physical activity.

This study had several limitations. Because of the qualitative nature of this study, it is not known whether the experiences and suggestions voiced by these participants would be similar to other young adults in other geographic areas. Participants were from one geographical region (northwest) and they were recruited from medical facilities; therefore the results may not be applied to other regions. The sample size was small with only 22 participants and there were more participants with type 1 diabetes than type 2 diabetes, so consequently participants were more concerned about blood glucose levels related to insulin. Participants provided limited responses when discussing physical activity or strategies to overcoming the barriers. Further research is needed to identify the lack of interest in this topic.

\section{Discussion}

Results from this focus group study identified that young adults, 18 to 28 years old, with either type 1or type 2 diabetes have unique challenges as they transition from pediatric to adult care, which has been previously recognized [1] , [4].The three themes on the perceived barriers to diabetes self-management: lack of understanding, lack of support, and lack of resources, discussed in this study have been previously noted [27].

To overcome lack of understanding, lack of support and lack of access participants recommended using the internet. The internet is an appropriate venue since approximately $77 \%$ of internet users looked for health information over the last year [28].In addition, other researchers [29] found that diabetes patients were more comfortable using the internet to seek information about a medical condition than they are contacting their doctor to share personal clinical information, to request disease-specific guidance and feedback, and to receive emotional support. Some educators [21] suggest that educational materials cover this age group's specific issues, e.g. hypoglycemia from binge drinking, relationships, and contraception. If health care providers and educators could find innovative ways of using the internet and cover age specific issues, young adult patients may be more inclined to listen to their suggestions.

In addition to providing information, the internet could also provide support for this age group through social networking with other diabetes patients and help them monitor their blood glucose. Approximately $92 \%$ of adults between the ages of 18-29 years use social networking sites [28].One outcome related to lack of support by family and friends was lapses in checking blood glucose. Cell phone glucose monitoring systems have been used as an effective way to get adolescents to monitor their blood glucose [30]. Newer technologies have helped people with type 1diabetes monitor their blood sugar levels daily and required fewer 
painful needle sticks [31].

\section{Conclusion}

The information gained from this study provides insight to the perceived barriers to diabetes management, which can be used to design education and support programs that meet the specific needs of the young adult. Further, social support networks were described as key components of educational programs and should be utilized to improve needed support. This study provides implications for health care providers on how they communicate and contact their patients. Young adults in this study liked and used internet applications. They also like having contact with their providers. New and innovative apps for patients with diabetes to share immediate information with their providers may have merit for this population.

Physical activity plays a pivotal role in diabetes management and well-being. Further research needs to be conducted with this age population to help them become more physically active and to customize treatment plans for young adults.

\section{Acknowledgements}

Lisa Galbraith, BS, RD; and Heather Blume, BS, RD, LD assisted in data collection and Rockwood Clinic, Kootenai Diabetes and Endocrinology Center, and Humphreys Diabetes Center who hosted the focus group meetings and assisted in participant recruitment.

This project was supported by NIH Challenge Grant Number 1RC1LM01065101.

Its contents are solely the responsibility of the authors and do not necessarily represent the official views of the NIH.

\section{REFERENCES}

[1] Centers for Disease Control and Prevention, "National diabetes fact sheet: National estimates and general information on diabetes and pre-diabetes in the United States," 2006, Online available from http://www.cdc.gov/diabetes/pubs/pdf/ndfs_2011.pd.

[2] American Diabetes Association, "Economic costs of diabetes in the U.S. in 2012," Diabetes Care, 2013, Online available from

http://care.diabetesjournals.org/content/early/2013/03/05/dc1 2-2625.full.pdf + html.

[3] M. Harris, K. Freeman, and D. Duke, "Transitioning from pediatric to adult health care: Dropping off the face of the earth," American Journal of Lifestyle Medicine, vol. 5, 85-91, 2011.

[4] M. Svensson, G. Sundkvist, H. Arnqvist, E. Björk, G. Blohmé, J. Bolinder, and J. Eriksson, "Signs of nephropathy may occur early in young adults with diabetes despite modern diabetes management," Diabetes Care, vol. 26, no. 10,2903-2909, 2003.

[5] M. Henricsson, L. Nystrőm, G. Blohmé, J.ő stman, C. Kullberg, M. Svensson, and G. Sunkvist, "The incidence of retinopathy 10 years after diagnosis in young adult people with diabetes," Diabetes Care,vol. 26, no. 2, 349-354, 2003.

[6] A. Maguire, M. Craig, A. Craighead, A. Chan, J. Cusumano, S. Hing and K. Donaghue, "Autonomic nerve testing predicts the development of complications, "Diabetes Care, vol. 30 no. 4, 77-82, 2007.

[7] S. Laing, A. Swerdlow, S. Slater, J. Botha, A. Burden, N. Waugh, and H. Keen, "The British Diabetic Association cohort study II: Cause-specific mortality in patients with insulin-treated diabetes mellitus," Diabetes Medicine, vol. 16, 466-471, 1999.

[8] B. Anderson, and H. Wolpert, "A developmental perspective on the challenges of diabetes education and care during the young adult period, "Patient Education and Counseling, vol. 53 , no. 3, 347-52,2004.

[9] G. Dovey-Pearce, R. Hurrell, C. May, C. Walker, and Y. Doherty, "Young adults' (16-25 years) suggestions for providing developmentally appropriate diabetes services: A qualitative study," Health and Social Care in the Community, vol. 13,409-419, 2005.

[10] [G. Dovey-Pearce, Y. Doherty, and C. May, "The influence of diabetes upon adolescent and young adult development: A qualitative study," British Journal of Health Psychology, vol. 12, 75-91, 2007.

[11] S. Mulvaney, E. Mudasiru, D. Schlundt, C. Baughman, M. Fleming, A. VanderWoude, and R. Rothman, "Self-management in type 2 diabetes: The adolescent perspective," The Diabetes Educator, vol. 34, no.4, 674-682, 2008 .

[12] M. Wdowik, P. Kendall, M. Harris, and G. Auld, "Expanded health belief model predicts diabetes self-management in college students, "Journal of Nutrition Education, vol. 33, no. $1,17-23,2001$

[13] R. Krueger, and M. Casey, "Focus groups: A practical guide for applied research (3rd ed.)," Sage Publications, Thousand Oaks, CA, 2001.

[14] Qualis Research Associates, "The Ethnograph v5.0. A program for the analysis of text based data," Sage Publications, London, 2000.

[15] K. Neuendorf, "The Content Analysis Guidebook," Sage Publicaitons, Thousand Oaks, CA, 2002.

[16] D. DeRidder, K. Schreurs, and R. Kuijer, "Is spousal support always helpful to patients with asthma or diabetes? A prospective study," Psychology \& Health, vol. 20, no.4, 497-508, 2005.

[17] I. Coyne, and P. Gallagher, "Participation in communication and decision-making: children and young people's experiences in a hospital setting," Journal of Clinical Nursing, vol. 20 , no.15, 2334-2343, 2011.

[18] M. Maryniuk, "The new shape of medical nutrition therapy," Diabetes Spectrum, vol. 13, no. 3, 122-124, 2000.

[19] T. Rintala, P. Jaatinen, E. Paavilainen, and P. 
Astedt-Kurki,"Interrelation between adult persons with diabetes and their family: A systematic review of the literature," Journal of Family Nursing, vol. 19, no. 1, 3-28, 2013.

[20] K. Mulcahy, M. Maryniuk, M. Peeples, M., Peyrot, D. Tomky, T. Weaver, and P. Yarborough, "Diabetes self-management education core outcome measures," The Diabetes Educator, vol. 29 , no.5, 768-803, 2003.

[21] H. Wolpert, and B. Anderson, "Young adults with diabetes need for a new treatment paradigm," Diabetes Care, vol. 24, no. $9,1513-1514,2001$.

[22] E. Mishler, J. Clark, J. Ingelfinger, and M. Simon,"The language of attentive patient care: A comparison of two medical interviews," Journal of General Internal Medicine, vol. 4, 325-335, 1989.

[23] K. Wikblad, "Patients' perspectives of diabetes care and education," Journal of Advanced Nursing, vol.16, 837-844, 1991.

[24] R. Anderson, and J. Fitzgerald, "The relationship between diabetes-related attitudes and patients' self-reported adherence," Diabetes Education, vol.19, 287-292, 1993.

[25] American Diabetes Association, "Standards of medical care in diabetes," Diabetes Care, vol. 31, no. 1, S12-54, 2008.
[26] B. Bradley, "Financial concerns about insulin pumps,"2006, Online available from http://www.diabeteshealth.com/read/2006/02/01/4527/financ ial-concerns-about-insulin-pumps.

[27] N. Ramchandani,J. Catney-Kiser, C. Alter,S. Brink,S. Yeager, W. Tamborlane, and S. Chipkin,"Self-reported factors that affect glycemic control in college students with type 1 diabetes," Diabetes Educator, vol. 26, no. 4,656-666, 2004.

[28] Pew Research Social Networking, "Internet \& American Life Project surveys August 2012," 2013, Online available fromhttp://www.pewinternet.org/Commentary/2012/March/ Pew-Internet-Social-Networking.

[29] J. Greene, N. Choudhry, E. Kilabuk, and W. Shrank, "Online social networking by patients with diabetes: A qualitative evaluation of communication with Facebook," Journal of General Internal Medicine, vol. 26, no. 3, 287-292, 2001.

[30] [30] A. Carroll, L. DiMeglio, S. Stein, and D. Marrero, "Using a cell phone-based glucose monitoring system for adolescent diabetes management," The Diabetes Educator, vol. 37, 59-66, 2011.

[31] H. Yeh, T. Brown, N. Maruthur, P. Ranasinghe, Z. Berger, Y. Suh, and S. Golden, "Comparative effectiveness and safety of methods of insulin delivery and glucose monitoring for Diabetes Mellitus: A systematic review and meta-analysis," Annals of Internal Medicine, vol. 157 no.5,336-347, 2012. 